\title{
Gate depletion of an InSb two-dimensional electron gas
}

M. M. Uddin, ${ }^{1}$ H. W. Liu, ${ }^{1,2,3, a)}$ K. F. Yang, ${ }^{1,2}$ K. Nagase, ${ }^{1,2}$ K. Sekine, ${ }^{1}$ C. K. Gaspe, ${ }^{4}$ T. D. Mishima, ${ }^{4}$ M.

B. Santos, ${ }^{4}$ and Y. Hirayama ${ }^{1,2,5, b)}$

${ }^{1}$ Department of Physics, Tohoku University, Sendai, Miyagi 980-8578, Japan

${ }^{2}$ ERATO Nuclear Spin Electronics Project, Sendai, Miyagi 980-8578, Japan

${ }^{3}$ State Key Laboratory of Superhard Materials and Institute of Atomic and Molecular Physics, Jilin University, Changchun 130012, People's Republic of China

${ }^{4}$ Homer L. Dodge Department of Physics and Astronomy, University of Oklahoma, 440 West Brooks, Norman, Oklahoma 73019-2061, USA

${ }^{5}$ WPI Research Center, Advanced Institute for Materials Research, Tohoku University, Sendai 980-8577, Japan

We investigated the gate control of a two-dimensional electron gas (2DEG) confined to InSb quantum wells with an $\mathrm{Al}_{2} \mathrm{O}_{3}$ gate dielectric formed by atomic layer deposition on a surface layer of $\mathrm{Al}_{0.1} \mathrm{In}_{0.9} \mathrm{Sb}_{0}$ InSb. The wider bandgap of $\mathrm{Al}_{0.1} \mathrm{In}_{0.9} \mathrm{Sb}$ compared to InSb resulted in a linear, sharp, and non-hysteretic response of the $2 \mathrm{DEG}$ density to gate bias in the structure with an $\mathrm{Al}_{0.1} \mathrm{In}_{0.9} \mathrm{Sb}$ surface layer. In contrast, a nonlinear, slow, and hysteretic (nonvolatile-memory-like) response was observed in the structure with an InSb surface layer. The $2 \mathrm{DEG}$ with the $\mathrm{Al}_{0.1} \mathrm{In}_{0.9} \mathrm{Sb}$ surface layer was completely depleted by application of a small gate voltage $(\sim-0.9 \mathrm{~V})$. 
A split-gate technique is now widely used for the fabrication of semiconductor nanostructures (e.g., quantum point contacts and quantum dots): a negative bias on surface gates locally depletes the underlying two-dimensional electron gas (2DEG) confined to a quantum well (QW), leaving electrons only in a desired region surrounded by gate areas. This technique has already been applied to most semiconductors ( $\mathrm{Si}$, GaAs, InAs, InGaAs, etc.), but not to the typical narrow-gap semiconductor InSb even though InSb is particularly appealing for spintronics applications (spin field-effect transistors, ${ }^{1-3}$ heat-driven spin devices, ${ }^{4}$ electron or nuclear-spin-based quantum bits, ${ }^{5,6}$ etc.) and for the detection of signatures of majorana Fermions due to strong spin-orbit coupling and giant $g$-factor. ${ }^{7}$ Because the relatively low barrier height of a Schottky contact to $(\mathrm{Al}) \mathrm{InSb}$ produces high current leakage, ${ }^{8,9}$ top gating with gate dielectrics ${ }^{10}$ becomes an attractive alternative for surface-gate fabrication of InSb QWs. However, several studies have highlighted difficulties in growing high quality gate dielectrics on (Al)InSb and in forming a good interface between the dielectric and (Al)InSb layers. ${ }^{3,8}$

More recently, we have grown a high quality $\mathrm{Al}_{2} \mathrm{O}_{3}$ gate dielectric on an InSb QW structure with an InSb surface layer (hereafter referred to as "sample 1") using atomic layer deposition (ALD). ${ }^{11}$ The Fermi level of this sample is tuned almost entirely across the band gap of InSb via gate bias. The good interface between the $\mathrm{Al}_{2} \mathrm{O}_{3}$ and $\mathrm{InSb}$ layers makes this possible, and allows us to study the importance of the layer sequence in gate controllability. However, the 2DEG in such a QW cannot be fully depleted because hole accumulation at the InSb surface layer screens the gate electric field. Based on a self-consistent Schrödinger-Poisson (SP) simulation, we predicted that the 2DEG would be depleted in an InSb QW with a wider-band-gap $\mathrm{Al}_{\mathrm{x}} \mathrm{In}_{1-\mathrm{x}} \mathrm{Sb}$ surface layer that is expected to prevent the hole accumulation. ${ }^{11}$ In this letter, we show experimental evidence that the $2 \mathrm{DEG}$ in an $\mathrm{InSb} \mathrm{QW}$ with an $\mathrm{Al}_{0.1} \mathrm{In}_{0.9} \mathrm{Sb}$ surface layer (sample 2) is completely depleted. Particularly noteworthy is that the $\mathrm{Al}_{0.1} \mathrm{In}_{0.9} \mathrm{Sb}$ layer also has the advantages of suppressing a parallel conduction channel and of keeping a relatively low interface trap density in the depletion process as revealed by our modified SP simulation. The success in gate depletion of the 2DEG enables the application of the split-gate technique to InSb QWs, which has significant implications for realizing InSb-based spintronics.

The layer structure of sample 2, shown on the bottom axis of Fig. 1(a), is the same as that of sample 1 except for the absence of an InSb cap layer and for a shorter distance between the second silicon $(\mathrm{Si}) \delta$ doped layer and the QW (15 $\mathrm{nm}$ for sample 1 and $10 \mathrm{~nm}$ for sample 2). After a Hall bar mesa $(80 \mu \mathrm{m} \times 30$ 
$\mu \mathrm{m})$ was defined by photolithography, a 40 -nm-thick $\mathrm{Al}_{2} \mathrm{O}_{3}$ layer was deposited using $\mathrm{ALD}$ at $130^{\circ} \mathrm{C}$ without any surface treatment ${ }^{12}$ (details of the ALD growth and the epitaxial layer structure are described in Ref. 11). The lower panel of Fig. 1 shows atomic force microscopy (AFM) images of ALD- $\mathrm{Al}_{2} \mathrm{O}_{3}$ grown on the InSb (sample 1) and $\mathrm{Al}_{0.1} \mathrm{In}_{0.9} \mathrm{Sb}$ (sample 2) surface layers with a root-mean-square (rms) roughness of $0.57 \mathrm{~nm}$ and $0.33 \mathrm{~nm}$, respectively, suggesting a smooth and dense insulator film for both samples. Indium was then evaporated for the Ohmic contacts and the top gate. We note that the following results were also obtained for a sample with $\mathrm{Al}_{2} \mathrm{O}_{3}$ grown by $\mathrm{ALD}$ at $150^{\circ} \mathrm{C}$. All experiments and simulations in this work were carried out at a temperature of $2 \mathrm{~K}$ unless noted otherwise. A standard AC lock-in technique (13.3 Hz; $35 \mathrm{nA})$ was used for both Hall and magnetoresistance (MR) measurements.

Figure 1(a) shows the energy band diagram (up to $85 \mathrm{~nm}$ in depth) of sample 2 at zero gate bias $\left(V_{\mathrm{g}}\right)$ calculated from the SP simulation with a Schottky barrier model. ${ }^{11,13}$ In this SP simulation, a unique fitting parameter is the Schottky barrier height $\phi_{\mathrm{B}}$ that is determined by properties of the semiconductor surface and interface states. The energy difference between the conduction band (CB) minimum $E_{\mathrm{c}}$ at zero depth and the Fermi energy $E_{\mathrm{F}}$ (set at $0 \mathrm{eV}$ ) corresponds to $\phi_{\mathrm{B}}$, which can be adjusted manually for agreement between the simulated and the measured 2DEG density $n_{\mathrm{s}}$. For instance, $\phi_{\mathrm{B}}=0.14 \mathrm{eV}$ in Fig. 1(a) gives $n_{\mathrm{s}}$ $=3.32 \times 10^{15} \mathrm{~m}^{-2}$, consistent with the Hall measurement result at $V_{\mathrm{g}}=0$. It is also seen that such a $\phi_{\mathrm{B}}$ locates the $\mathrm{CB}$ discontinuity at the $\mathrm{Al}_{0.1} \mathrm{In}_{0.9} \mathrm{Sb} / \mathrm{Al}_{0.2} \mathrm{In}_{0.8} \mathrm{Sb}$ interface a little above $E_{\mathrm{F}}$, thus preventing the formation of a parallel conduction channel at the discontinuity. ${ }^{11}$ This is evidenced by the zero-bias MR plot in the upper inset to Fig. 1(b): the MR property is similar to that of a single 2DEG except for the presence of non-zero longitudinal resistance $R_{\mathrm{xx}}$ under the Hall $\left(R_{\mathrm{xy}}\right)$ plateau, indicating that the parallel channel is strongly suppressed. Note that the parallel channel emerges at a small $V_{\mathrm{g}} \sim 0.05 \mathrm{~V}$ (data not shown) because the positive bias will lower the $\mathrm{CB}$ discontinuity below $E_{\mathrm{F}}$. More importantly, the relatively wide band gap $(\sim 0.42 \mathrm{eV})^{14}$ of $\mathrm{Al}_{0.1} \mathrm{In}_{0.9} \mathrm{Sb}$ in sample 2 keeps the valence band (VB) maximum $E_{\mathrm{v}}$ at zero depth far from $E_{\mathrm{F}}$ as shown in Fig. 1(a), which avoids hole accumulation at the surface under negative bias ${ }^{11}$ and thereby enables complete depletion of the 2DEG. Fig. 1(b) shows the $V_{\mathrm{g}}$ dependence of $n_{\mathrm{s}}$ and the mobility $(\mu)$ of sample 2, obtained from Hall and resistivity measurements. Apparently, both $n_{\mathrm{s}}$ and $\mu$ decrease with increasing $\left|V_{\mathrm{g}}\right|$. The data at $V_{\mathrm{g}}=-0.6 \mathrm{~V}$ gives $R_{\mathrm{xx}} \sim 23 \mathrm{k} \Omega$ at zero magnetic field (see also the MR curve in Fig. 1(b), lower inset). A further increase of $\left|V_{\mathrm{g}}\right|$ greatly enhances $R_{\mathrm{xx}}$ and thus invalidates the Hall measurement, as expected when both $n_{\mathrm{s}}$ and $\mu$ become sufficiently small. The 
intersection of the $n_{\mathrm{s}}-V_{\mathrm{g}}$ plot with the horizontal axis at $n_{\mathrm{s}}=0$ in Fig. 1(b) allows us to estimate a pinch-off voltage of $V_{\mathrm{p}} \sim-0.9 \mathrm{~V}$ for complete depletion of the 2DEG. We should mention that $\left|V_{\mathrm{p}}\right|$ is much smaller than a threshold voltage of $4 \mathrm{~V}$ for current leakage through the $\mathrm{ALD}$-grown $\mathrm{Al}_{2} \mathrm{O}_{3}$ in this sample. Thus, gate depletion of the InSb 2DEG has been demonstrated in sample 2 .

Besides its role in gate depletion of the InSb $2 \mathrm{DEG}$ discussed above, the $\mathrm{Al}_{0.1} \mathrm{In}_{0.9} \mathrm{Sb}$ surface layer is also found to form a good interface with the ALD-grown $\mathrm{Al}_{2} \mathrm{O}_{3}$ layer. For comparison, the $n_{\mathrm{s}}-V_{\mathrm{g}}$ plots of sample 1 and sample 2 are shown in Fig. 2(a). It is clear that the change of $n_{\mathrm{s}}$ in response to $V_{\mathrm{g}}$ is different for the two samples. Firstly, $n_{\mathrm{s}}$ of sample 1 decreases slowly and nonlinearly with increasing $\left|V_{\mathrm{g}}\right|$ and finally tends to saturate, while that of sample 2 decreases rapidly and almost linearly with increasing $\left|V_{\mathrm{g}}\right|$, resulting in a large slope of $\mathrm{d} n_{\mathrm{s}} / \mathrm{d} V_{\mathrm{g}}=3.9 \times 10^{15} \mathrm{~m}^{-2} \mathrm{~V}^{-1}$. Secondly, a notable hysteresis is observed in sample 1 but only a very small one appears in sample 2 . We applied both an equivalent capacitance model and a SP simulation to explain these differences. The inset to Fig. 2(a) depicts an equivalent capacitance circuit for sample 2, where $C_{\mathrm{ox}}$ and $C_{\mathrm{sc}}$ are the capacitance per unit area of the $\mathrm{Al}_{2} \mathrm{O}_{3}$ and semiconductor layers above the QW, respectively, and $C_{\mathrm{it}}=e^{2} D_{\mathrm{it}}$ ( $e$ is the electron charge and $D_{\mathrm{it}}$ is the interface trap density) is the capacitance associated with the interface traps ${ }^{15}$ and $C_{2 \mathrm{D}}=e^{2} m^{*} m_{e} / \pi \hbar^{2}\left(m^{*} \sim 0.0135\right.$ is the effective mass of InSb, $m_{\mathrm{e}}$ is the electron mass, and $\hbar$ is Planck's constant divided by $2 \pi$ ) is the quantum capacitance $^{16}$ of the InSb 2DEG. In the ideal case of $C_{\mathrm{it}}=0$, the $n_{\mathrm{s}}-V_{\mathrm{g}}$ dependence is given by $e n_{\mathrm{s}}=C_{\mathrm{tot}} V_{\mathrm{g}}$ ( $C_{\text {tot }}$ is the total capacitance of $C_{\mathrm{ox}}, C_{\mathrm{sc}}$ and $C_{2 \mathrm{D}}$ ), as indicated by a dashed line in Fig. 2(a). The slope $\mathrm{d} n_{\mathrm{s}} / \mathrm{d} V_{\mathrm{g}} \sim 5.5 \times 10^{15} \mathrm{~m}^{-2} \mathrm{~V}^{-1}$ of this line is close to that of sample 2, indicating a good interface between the ALD-grown $\mathrm{Al}_{2} \mathrm{O}_{3}$ and the $\mathrm{Al}_{0.1} \mathrm{In}_{0.9} \mathrm{Sb}$ layer. In the case of $C_{\mathrm{it}} \neq 0, \mathrm{~d} n_{\mathrm{s}} / \mathrm{d} V_{\mathrm{g}}=\left[1+C_{\mathrm{it}}\left(C_{\mathrm{sc}}+\right.\right.$ $\left.\left.C_{2 \mathrm{D}}\right) /\left(C_{\mathrm{sc}} C_{2 \mathrm{D}}\right)\right]^{-1} C_{\mathrm{tot}}^{\prime} / e\left(C_{\mathrm{tot}}^{\prime}\right.$ is the total capacitance of $C_{\mathrm{ox}}, C_{\mathrm{sc}}, C_{\mathrm{it}}$ and $\left.C_{2 \mathrm{D}}\right)$ is deduced from the equivalent circuit and used to fit our data, giving a relatively low $D_{\mathrm{it}}=1.05 \times 10^{16} \mathrm{~m}^{-2} \mathrm{eV}^{-1}$. However, this capacitance model is only appropriate for the data analysis at $-1.6 \mathrm{~V} \leq V_{\mathrm{g}} \leq-1.2 \mathrm{~V}$ in sample 1 because it is difficult to account for the effects of the parallel conduction channel at $V_{\mathrm{g}}>-1.2 \mathrm{~V}$ and hole accumulation at $V_{\mathrm{g}}<-1.6 \mathrm{~V}$ (see below) in the equivalent circuit. The value of $\mathrm{d} n_{\mathrm{s}} / \mathrm{d} V_{\mathrm{g}}=6 \times 10^{14} \mathrm{~m}^{-2} \mathrm{~V}^{-1}$ at $-1.6 \mathrm{~V} \leq V_{\mathrm{g}} \leq-$ $1.2 \mathrm{~V}$ (downward sweep) yields a high $D_{\mathrm{it}}=1.7 \times 10^{17} \mathrm{~m}^{-2} \mathrm{eV}^{-1}$. Although $D_{\mathrm{it}}$ of the two samples is quite different, care should be taken in concluding that the interface of $\mathrm{Al}_{2} \mathrm{O}_{3} / \mathrm{Al}_{0.1} \mathrm{In}_{0.9} \mathrm{Sb}$ is better than that of $\mathrm{Al}_{2} \mathrm{O}_{3} / \mathrm{InSb}$ because $D_{\text {it }}$ is energy dependent, as discussed below. 
Fig. 2(b) shows the $V_{\mathrm{g}}$ dependence of $E_{\mathrm{F}}$ obtained from the SP simulation. Since $E_{\mathrm{F}}$ is fixed at $0 \mathrm{eV}$ in the simulation, the modification of $E_{\mathrm{F}}$ is reflected in the movement of $E_{\mathrm{v}}$ relative to $E_{\mathrm{F}}$. The energy difference $E_{\mathrm{F}}-E_{\mathrm{v}}$ is calculated by $E_{\mathrm{g}}-E_{\mathrm{c}}$, where $E_{\mathrm{g}}$ is the band gap of the semiconductor surface layer and $E_{\mathrm{c}}$ is determined in the same way as $\phi_{\mathrm{B}}$. It is shown in Fig. 2(b) that $E_{\mathrm{F}}$ in sample 2 is readily tuned by a small bias, indicating a very weak pinning of $E_{\mathrm{F}}$. Because our SP simulation is invalid in the presence of a parallel channel or hole accumulation, there are only two data points for sample 1 . The data point at $V_{\mathrm{g}}=-$ $1.6 \mathrm{~V}$ suggests that $E_{\mathrm{v}}$ touches the Fermi level. As $\left|V_{\mathrm{g}}\right|$ is further increased, holes accumulate at the semiconductor surface and screen the external electric field, which accounts for the saturation of $n_{\mathrm{s}}$ in Fig. 2(a). On the other hand, $E_{\mathrm{F}}-E_{\mathrm{v}}$ of sample 2 at $V_{\mathrm{p}}=-0.9 \mathrm{~V}$ (solid symbol) is greater than zero, indicating that $n_{\mathrm{s}}$ continues to decrease with increasing $\left|V_{\mathrm{g}}\right|$ until depletion. In order to analyze the interface trap states, we extend the SP simulation to include a model of metal-oxide-semiconductor (MOS) structures. In this modified SP simulation, the $V_{\mathrm{g}}$ applied to the metal causes voltage drops across the oxide layer and at the semiconductor surface. The voltage $\left|V_{\mathrm{ox}}\right|$ across the oxide is equal to $\left|V_{\mathrm{g}}\right|-E_{\mathrm{c}} / \mathrm{e}$, neglecting any workfunction difference and interfacial layer thickness. The Gauss law then gives $Q_{\mathrm{it}}=\varepsilon_{0} \varepsilon_{\mathrm{ox}} E_{\mathrm{ox}}-\varepsilon_{0} \varepsilon_{\mathrm{sc}} E_{\mathrm{sc}}$ (electric field $E_{\mathrm{ox}}=V_{\mathrm{ox}} / d_{\mathrm{ox}}$ with $\mathrm{Al}_{2} \mathrm{O}_{3}$ thickness $d_{\mathrm{ox}}=40 \mathrm{~nm}$, electric field $E_{\mathrm{sc}}$ at the semiconductor surface obtained from the SP simulation). Based on the equation $\mathrm{d} Q_{\mathrm{it}} / \mathrm{d}\left(E_{\mathrm{F}}-E_{\mathrm{v}}\right)=-\mathrm{e} D_{\mathrm{it}},{ }^{15}$ we obtain the energy dependent $D_{\mathrm{it}}$ of the two samples shown in the inset to Fig. 2(b). We see that $D_{\text {it }}$ around the midgap $(0.21 \mathrm{eV})$ of $\mathrm{Al}_{0.1} \mathrm{In}_{0.9} \mathrm{Sb}$ in sample 2 is about $1 \times 10^{16} \mathrm{~m}^{-2} \mathrm{eV}^{-1}$, consistent with the result of the capacitance model. It is clear that a low $D_{\mathrm{it}}$ is kept in the depletion process, accounting for a low-voltage gate operation. Because $D_{\text {it }}$ around the midgap $(0.118 \mathrm{eV})$ of InSb in sample 1 cannot be calculated due to the presence of a parallel channel, we cannot compare the interfacial properties of the two samples. Nevertheless, the data point indicating $D_{\mathrm{it}} \sim 1.6 \times 10^{17} \mathrm{~m}^{-2} \mathrm{eV}^{-1}$ near the valence band of sample 1 agrees with the capacitance model calculation.

We now discuss the density hysteresis observed in sample 1 . This hysteresis is found to behave like a nonvolatile memory, as shown in Fig. 3: it depends on the sweep direction and range of $V_{\mathrm{g}}$ but is nearly independent of the sweep rate, which is different from the observations in InAs/AlGaSb ${ }^{17}$ or $\mathrm{HgTe} / \mathrm{HgCdTe}^{18}$ QWs. Such a hysteretic behaviour is reminiscent of the capacitance-voltage $(C-V)$ characteristic of nonvolatile $\mathrm{Al}_{2} \mathrm{O}_{3}$ memory devices, where charge traps in a nonstoichiometric $\mathrm{Al}_{2} \mathrm{O}_{3}$ layer dominate the memory effect. ${ }^{19}$ Here we also assign charge traps in the $\mathrm{Al}_{2} \mathrm{O}_{3}$ layer to account for our 
hysteresis. As is shown in Fig. 3, the hysteresis becomes more prominent at more negative bias, where $D_{\text {it }}$ is large and holes accumulate at the semiconductor surface layer as discussed above. We expect that the oxygen vacancies in the $\mathrm{Al}_{2} \mathrm{O}_{3}$ layer with charge-state switching levels ${ }^{20}$ near the bandgap of InSb might act as charge traps: the fully ionized oxygen vacancies trap (detrap) electrons (holes) in the downward sweep of $V_{\mathrm{g}}$ and the neutral ones detrap (trap) electrons (holes) in the upward sweep of $V_{\mathrm{g}}$ via a tunnelling process. The stored charge $Q_{\mathrm{tr}}$ in the oxide, proportional to $D_{\mathrm{it}}$, the hole density $Q_{\mathrm{h}}$ and the oxygen vacancy density, induces a voltage drop that determines the hysteresis width. A large change in $V_{\mathrm{g}}$ leads to a large change in $Q_{\mathrm{h}}$ and $D_{\text {it }}$, accordingly resulting in a large hysteresis. Note that such a hysteresis can be observed up to $100 \mathrm{~K}$.

In conclusion, we have demonstrated gate depletion of the $2 \mathrm{DEG}$ in InSb QWs with an $\mathrm{Al}_{0.1} \mathrm{In}_{0.9} \mathrm{Sb}$ surface layer. This surface layer forms an excellent interface with an $\mathrm{Al}_{2} \mathrm{O}_{3}$ gate dielectric layer grown by ALD at low temperature $\left(130^{\circ} \mathrm{C}\right)$ in the absence of any surface treatment, leading to excellent gate performance characterized by low-voltage operation and negligible hysteresis. Following this development, the design and fabrication of split-gate-defined InSb quantum point contacts are now in progress.

M.M.U. would like to thank IIARE, WPI-AIMR, Tohoku University for S-SDC and RA fellowship. H.W.L. thanks the Program for New Century Excellent Talents of the University in China. 


\section{${ }^{a)}$ Electronic email: 1iuhw@m.tohoku.ac.jp \\ ${ }^{\text {b)} E l e c t r o n i c ~ e m a i l: ~ h i r a y a m a @ m . t o h o k u . a c . j p ~}$}

\section{References}

${ }^{1}$ I. Žutić, J. Fabian, and S. Das Sarma, Rev. Mod. Phys. 76, 323 (2004).

${ }^{2}$ G. A. Khodaparast, R. C. Meyer, X. H. Zhang, T. Kasturiarachchi, R. E. Doezema, S. J. Chung, N. Goel, M. B. Santos, Y. J. Wang, Physica E 20, 386 (2004).

${ }^{3}$ A. M. Gilbertson, W. R. Branford, M. Fearn, L. Buckle, P. D. Buckle, T. Ashley, and L. F. Cohen, Phys. Rev. B 79, 235333 (2009).

${ }^{4}$ C. M. Jaworski, R. C. Myers, E. Johnston-Halperin, and J. P. Heremans, Nature 487, 210 (2012).

${ }^{5}$ H. W. Liu, K. F. Yang, T. D. Mishima, M. B. Santos, and Y. Hirayama, Phys. Rev. B 82, 241304(R) (2010); K. F. Yang, H. W. Liu, K. Nagase, T. D. Mishima, M. B. Santos, and Y. Hirayama, Appl. Phys. Lett. 98, 142109 (2011); K. F. Yang, H. W. Liu, T. D. Mishima, M. B. Santos, K. Nagase, and Y. Hirayama, New J. Phys. 13, 083010 (2011); H. W. Liu, K. F. Yang, T. D. Mishima, M. B. Santos, and Y. Hirayama, Acta Phys. Sin. 61, 147302 (2012).

${ }^{6}$ H. A. Nilsson, P. Caroff, C. Thelander, M. Larsson, J. B. Wagner, L. Erik Wernersson, L. Samuelson, and H. Q. Xu, Nano Lett. 9, 3151 (2009); S. Nadj-Perge, V. S. Pribiag, J. W. G. van den Berg, K. Zou, S. R. Plissard, E. P. A. M. Bakkers, S. M. Frolov, and L. P. Kouwenhoven, Phys. Rev. Lett. 108, 166801 (2012); J. W. G. van den Berg, S. Nadj-Perge, V. S. Pribiag, S. R. Plissard, E. P. A. M. Bakkers, S. M. Frolov, and L. P. Kouwenhoven, Phys. Rev. Lett. 110, 066806 (2013).

${ }^{7}$ M. T. Deng, C. L. Yu, G. Y. Huang, M. Larsson, P. Caroff, and H. Q. Xu, Nano Lett. 12, 6414 (2012); V. Mourik, K. Zuo, S. M. Frolov, S. R. Plissard, E. P. A. M. Bakkers, L. P. Kouwenhoven, Science 336, 1003 (2012).

${ }^{8}$ J. K. Brask, S. Datta, M. L. Doczy, J. M. Blackwell, M. V. Metz, J. T. Kavalieros, and R. S. Chau, U.S. Patent No. 7,485,503 (2009).

${ }^{9}$ J. M. S. Orr, P. D. Buckle, M. Fearn, P. J. Wilding, C. J. Bartlett, M. T. Emeny, L. Buckle, and T. Ashley, Semicond. Sci. Technol. 21, 1408 (2006).

${ }^{10}$ S. Oktyabrsky and P. D. Ye, Fundamentals of III-V Semiconductor MOSFETs (Springer, New York, 2010). 
${ }^{11}$ M. M. Uddin, H. W. Liu, K. F. Yang, K. Nagase, T. D. Mishima, M. B. Santos, and Y. Hirayama, Appl. Phys. Lett. 101, 233503 (2012).

${ }^{12}$ C. H. Hou, M. C. Chen, C. H. Chang, T. B. Wu, C. D. Chiang, and J. J. Luo, J. Electrochem. Soc. 155, G180 (2008).

${ }^{13}$ S. Birner, Ph. D. thesis, Technical University of Munich, Germany, 2011.

${ }^{14}$ N. Dai, F. Brown, P. Barsic, G. A. Khodaparast, R. E. Doezema, M. B. Johnson, S. J. Chung, K. J. Goldmmer, and M. B. Santos, App. Phys. Lett. 73, 1101 (1998).

${ }^{15}$ S. M. Sze and K. K. Ng, Physics of Semiconductor Devices, 3rd ed. (John Wiley \& Sons, New York, 2007).

${ }^{16}$ S. Luryi, Appl. Phys. Lett. 52, 501 (1988).

${ }^{17}$ K. Suzuki, Y. Harada, F. Maeda, K. Onomitsu, T. Yamaguchi, and K. Muraki, Appl. Phys. Express 4, $125702(2011)$.

${ }^{18}$ J. Hinz, H. Buhmann, M. Schäfer, C. R. Becker, and L. W. Molenkamp, Semicond. Sci. Technol. 21, 501 (2006).

${ }^{19}$ S. Nakata, K. Saito, and M. Shimada, Appl. Phys. Lett. 87, 223110 (2005).

${ }^{20}$ M. Choi, A. Janotti, and C. G. Van de Walle, J. Appl. Phys. 113, 044501 (2013). 
FIG. 1. (Color online) (a) Band profile of sample 2 vs. depth along the growth direction at zero bias calculated from a self-consistent Schrödinger-Poisson simulation. The Fermi energy $E_{\mathrm{F}}$ is fixed at $0 \mathrm{eV}$, and $E_{\mathrm{c}}$ and $E_{\mathrm{v}}$ denote the conduction-band minimum and the valence-band maximum, respectively. The $\mathrm{Si}$ $\delta$-doped regions in the $\mathrm{Al}_{\mathrm{x}} \mathrm{In}_{1-\mathrm{x}} \mathrm{Sb}$ layers (see bottom axis) are indicated by vertical arrows. (b) Electron density $n_{\mathrm{s}}$ and mobility $\mu$ of the InSb 2DEG as a function of gate bias $V_{\mathrm{g}}$ for sample 2 . The dash-dotted line points to the pinch-off voltage $V_{\mathrm{p}}$. Insets show the magnetic-field $(B)$ dependent longitudinal resistance $R_{\mathrm{xx}}$ and Hall resistance $R_{\mathrm{xy}}$ at $V_{\mathrm{g}}=0 \mathrm{~V}$ and $-0.6 \mathrm{~V}$. Lower panel: AFM images of the ALD-grown $\mathrm{Al}_{2} \mathrm{O}_{3}$ of sample 1 and sample 2.

FIG. 2. (Color online) (a) $n_{\mathrm{s}}-V_{\mathrm{g}}$ plots of sample 1 and sample 2 . Arrows indicate the gate sweep direction. Inset shows an equivalent capacitance circuit of the gated $\mathrm{InSb} \mathrm{QW}$. The symbol $C_{\mathrm{x}}$ denotes the capacitance per unit area. The calculation of $C_{\mathrm{ox}(\mathrm{sc})}=\varepsilon_{\mathrm{ox}(\mathrm{sc})} \varepsilon_{0} / d_{\mathrm{ox}(\mathrm{sc})}\left(\varepsilon_{0}\right.$ is vacuum permittivity) is based on the following parameters: dielectric constant $\varepsilon\left(\mathrm{Al}_{2} \mathrm{O}_{3}: 7 ; \mathrm{InSb}: 16.82 ; \mathrm{Al}_{0.1} \mathrm{In}_{0.9} \mathrm{Sb}: 16.34 ; \mathrm{Al}_{0.2} \mathrm{In}_{0.8} \mathrm{Sb}: 15.86\right)$ and thickness $d$ in units of $\mathrm{nm}\left(\mathrm{Al}_{2} \mathrm{O}_{3}: 40 ; \mathrm{InSb}: 10 ; \mathrm{Al}_{0.1} \mathrm{In}_{0.9} \mathrm{Sb}: 30 ; \mathrm{Al}_{0.2} \mathrm{In}_{0.8} \mathrm{Sb}: 20\right)$. The $n_{\mathrm{s}}-V_{\mathrm{g}}$ dependence of the capacitance circuit with $C_{\mathrm{it}}=0$ is indicated by the dashed line. (b) $V_{\mathrm{g}}$ dependence of $E_{\mathrm{F}}-E_{\mathrm{v}}$ calculated from the SP simulation. The open symbols are calculations for the data in Fig. 2(a) (downward sweep) and the solid one is for the data at $V_{\mathrm{p}}=-0.9 \mathrm{~V}$ in Fig. 1(b). The dashed line is drawn to guide the eye. Inset shows the interface trap density $D_{\mathrm{it}}$ as a function of $E_{\mathrm{F}}-\bar{E}_{\mathrm{V}}$ obtained from our modified SP simulation. $\bar{E}_{\mathrm{V}}$ is the average value of $E_{\mathrm{F}}-E_{\mathrm{v}}$ at neighbouring $V_{\mathrm{g}}$.

FIG. 3. (Color online) Hysteresis in the $n_{\mathrm{s}}-V_{\mathrm{g}}$ plot of sample 1 as a function of gate sweep direction and range. The solid (dashed) line denotes the upward (downward) sweep. 


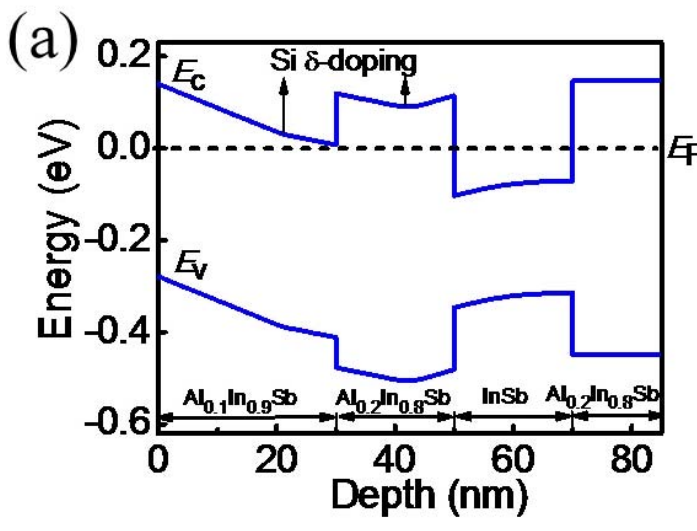

sample 1

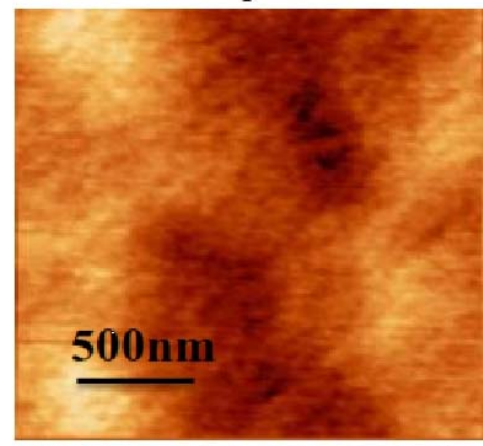

$3 n m$

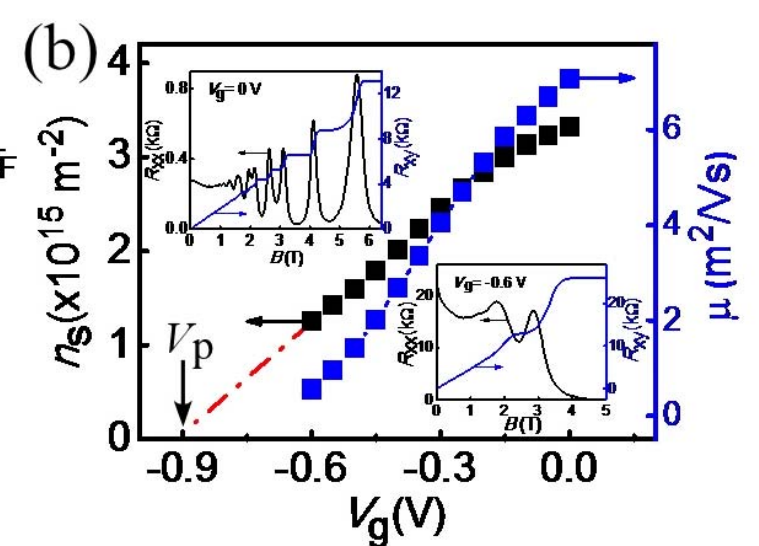

sample 2

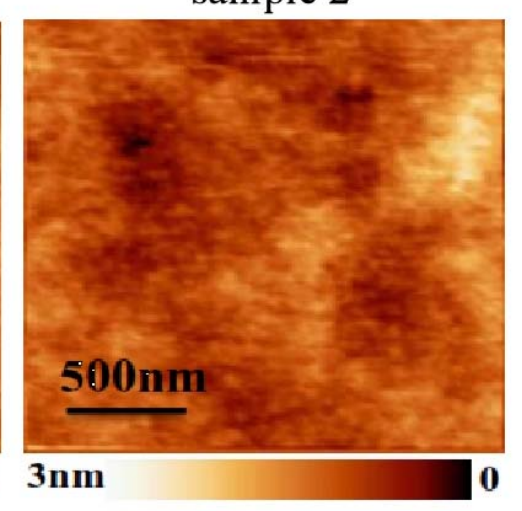

FIG. 1 

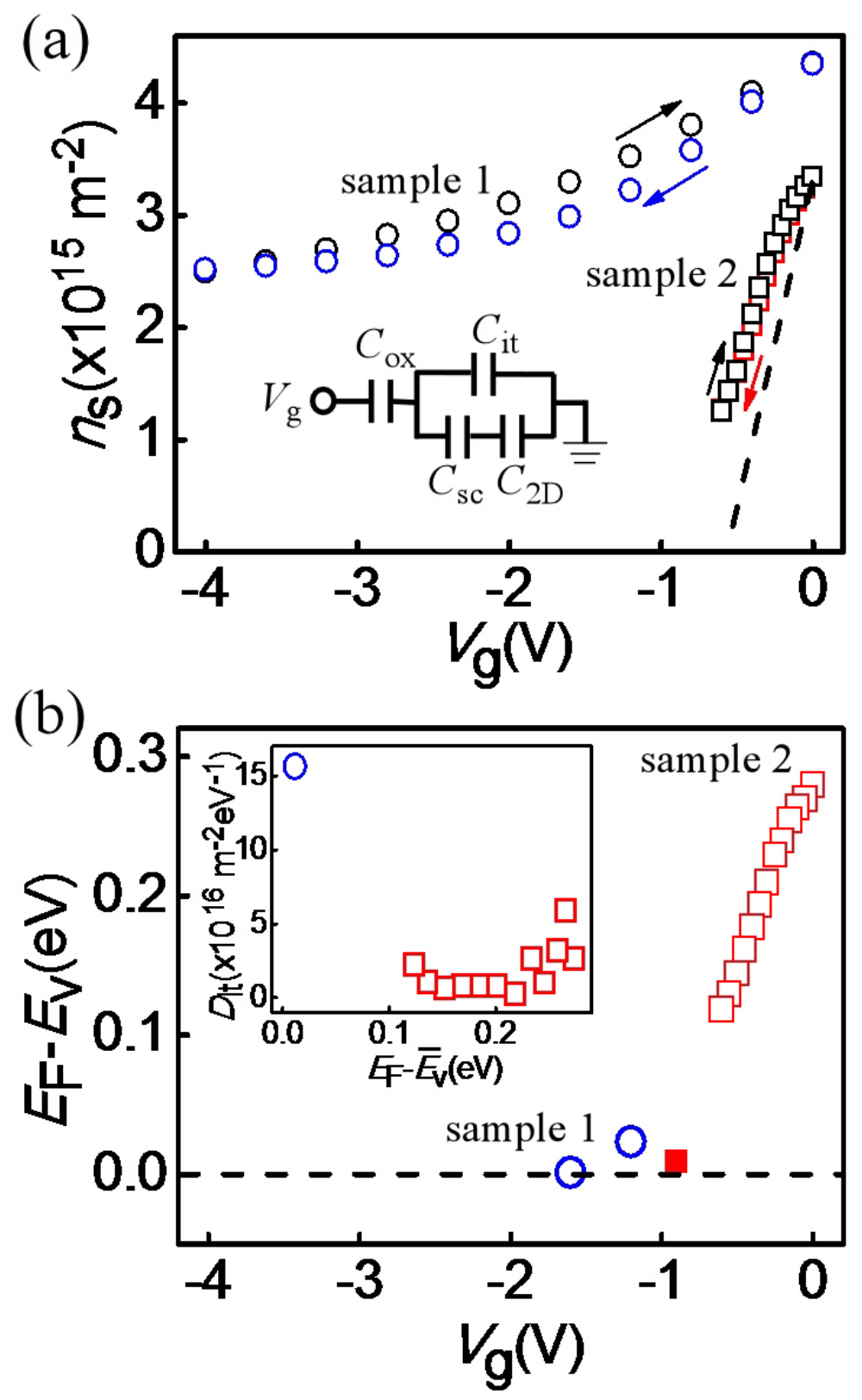

FIG. 2 


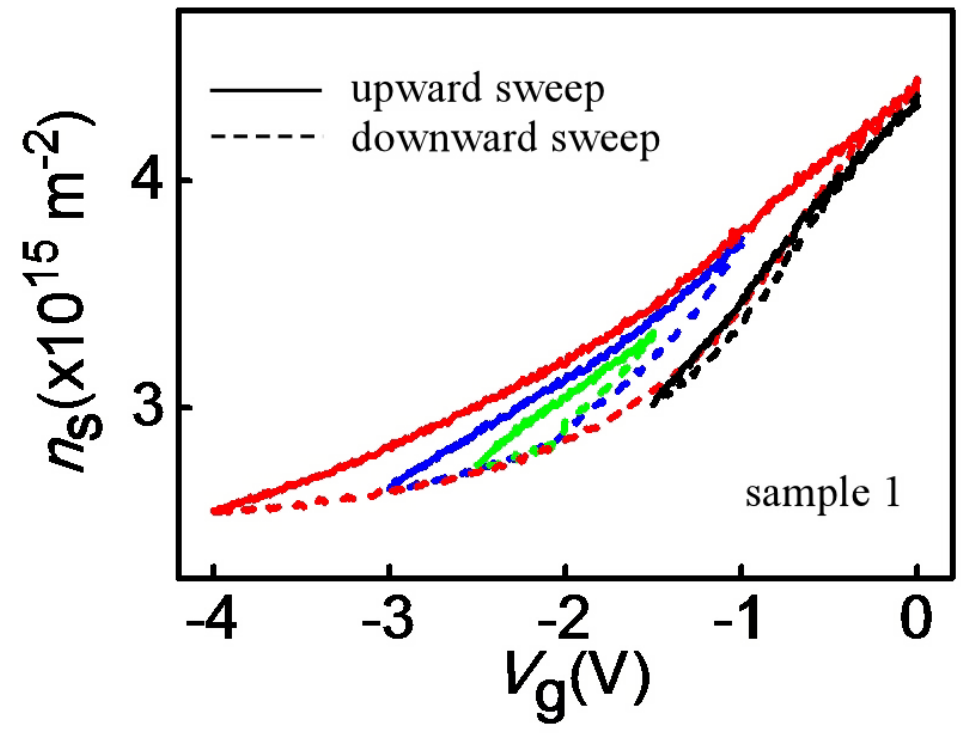

FIG. 3 